DESY $\quad 09-227$

\title{
Reconstruction of Baxter Q-operator from Sklyanin SOV for cyclic representations of integrable quantum models
}

G. Niccoli

DESY, Notkestr. 85, 22603 Hamburg, Germany

\begin{abstract}
In [1], the spectrum (eigenvalues and eigenstates) of a lattice regularizations of the SineGordon model has been completely characterized in terms of polynomial solutions with certain properties of the Baxter equation. This characterization for cyclic representations has been derived by the use of the Separation of Variables (SOV) method of Sklyanin and by the direct construction of the Baxter Q-operator family. Here, we reconstruct the Baxter Q-operator and the same characterization of the spectrum by only using the SOV method. This analysis allows us to deduce the main features required for the extension to cyclic representations of other integrable quantum models of this kind of spectrum characterization.
\end{abstract}




\section{Introduction}

The integrability of a quantum model is by definition related to the existence of a mutually commutative family $\mathcal{Q}$ of self-adjoint operators $T$ such that

$$
\begin{aligned}
& \text { (A) }\left[\mathrm{T}, \mathrm{T}^{\prime}\right]=0, \quad \forall \mathrm{T}, \mathrm{T}^{\prime} \in \mathcal{Q}, \\
& \text { (B) }[\mathrm{T}, \mathrm{U}]=0, \quad \forall \mathrm{T} \in \mathcal{Q}, \\
& \text { (C) if }[\mathrm{T}, \mathrm{O}]=0, \quad \forall \mathrm{T} \in \mathcal{Q}, \quad \text { then } \mathrm{O}=\mathrm{O}(\mathcal{Q}),
\end{aligned}
$$

where $\mathrm{U}$ is the unitary operator defining the time-evolution in the model; note that the property (C) stays for the completeness of the family $\mathcal{Q}$. In the framework of the quantum inverse scattering method [2, 3, 4] the Lax operator $L(\lambda)$ is the mathematical tool which allows to define the transfer matrix:

$$
\mathrm{T}(\lambda)=\operatorname{tr}_{\mathbb{C}^{2}} \mathrm{M}(\lambda), \quad \mathrm{M}(\lambda) \equiv\left(\begin{array}{ll}
\mathrm{A}(\lambda) & \mathrm{B}(\lambda) \\
\mathrm{C}(\lambda) & \mathrm{D}(\lambda)
\end{array}\right) \equiv L_{\mathrm{N}}(\lambda) \ldots L_{1}(\lambda)
$$

a one parameter family of mutual commutative self-adjoint operators. The integrability of the model follows from $\mathrm{T}(\lambda)$ if the properties (B) and (C) of definition (1.1) can be proven for it. In some quantum model the integrability is derived by proving the existence of a further one-parameter family of self-adjoint operators the Q-operator which by definition satisfies the following properties:

$$
[\mathrm{Q}(\lambda), \mathrm{Q}(\mu)]=0, \quad[\mathrm{~T}(\lambda), \mathrm{Q}(\mu)]=0, \quad \forall \lambda, \mu \in \mathbb{C}
$$

plus the Baxter equation with the transfer matrix:

$$
\mathrm{T}(\lambda) \mathrm{Q}(\lambda)=\mathrm{a}(\lambda) \mathrm{Q}\left(q^{-1} \lambda\right)+\mathrm{d}(\lambda) \mathrm{Q}(q \lambda) .
$$

This is in particular the case for those models (like Sine-Gordon [1]) for which the time-evolution operator $\mathrm{U}$ is expressed in terms of $\mathrm{Q}$. A natural question arises: Is the integrable structure of these quantum models completely characterized by the transfer matrix $\mathrm{T}(\lambda)$ ?

Note that a standard procedure 1 to prove the existence of $Q(\lambda)$ is by a direct construction of an operator solution of the Baxter equation (1.4). Moreover, the coefficients a $(\lambda)$ and $d(\lambda)$ as well as the analytic and asymptotics properties of $\mathrm{Q}(\lambda)$ are some model dependent features which are derived by the construction. Let us recall that the general strategy [11, 12, 13, 14, 15] of this construction is to find a gauge transformation 2 such that the action of each gauge transformed Lax matrix on $Q(\lambda)$ becomes upper-triangular. Then the Q-operator assumes a factorized local form and the problem of its existence in such a form is reduced to the problem of the existence of some model dependent special function?

\footnotetext{
${ }^{1}$ It is worth recalling that there are also others constructions of the Q-operator. An interesting example is presented in the series of works [5, 6, 7] by V.V. Bazhanov, S.L. Lukyanov and A.B. Zamolodchikov on the integrable structure of conformal field theories. In [6 7] the Q-operator is obtained as a transfer-matrix by a trace procedure of a fundamental $L$-operator with $q$-oscillator representation for the auxiliary space (see also [8] 9]). This construction can be extended to massive integrable quantum field theories as it was argued by the same authors in [10].

${ }^{2}$ It leaves unchanged the transfer matrix while modifies the monodromy matrix $M(\lambda)$ defined in 1.2 .

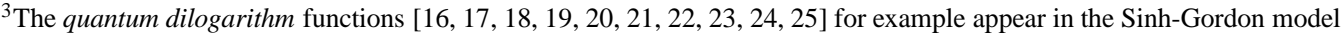
[26], in their non-compact form, and in the Sine-Gordon model [1], in their cyclic form.
} 
It is worth pointing out that on the one hand the construction of these special functions for general models can represent a concrete technical problem 4 and that on the other hand the existence of such functions is only a sufficient criterion for the existence of $Q(\lambda)$. It is then a relevant question if it is possible to bypass this kind of construction providing a different proof of the existence of $Q(\lambda)$.

Given an integrable quantum model the first fundamental task to solve is the exact solution of its spectral problem, i.e. the determination of the eigenvalues and the simultaneous eigenstates of the operator family $\mathcal{Q}$, defined in (1.1). There are several methods to analyze this spectral problem as the coordinate Bethe ansatz [27, 28, 29], the TQ method [28], the algebraic Bethe ansatz (ABA) [2, 3, 4], the analytic Bethe ansatz [30] and the separation of variables (SOV) method of Sklyanin [31, 32, 33]; this last one seems to be more promising. Indeed, on the one hand it resolves the problems related to the reduced applicability of other methods (like ABA) and on the other hand it directly implies the completeness of the characterization of the spectrum which instead for other methods has to be proven.

For cyclic representations [34] of integrable quantum models the SOV method should lead to the characterization of the eigenvalues and the simultaneous eigenstates of the transfer matrix $T(\lambda)$ by a finit 5 system of Baxter-like equations. However, it is worth pointing out that such a characterization of the spectrum is not the most efficient; this is in particular true in view of the analysis of the continuum limit. Here the main question reads: Is it possible to define a set of conditions under which the SOV characterization of the spectrum can be reformulated in terms of a functional Baxter equation? In fact, this is equivalent to ask if we can reconstruct the Q-operator from the finite system of Baxter-like equations. In this case the solution of the spectral problem is reduced to the classification of the solutions of the Baxter equation which satisfy some analytic and asymptotic properties fixed by the operators $T$ and $Q$.

The lattice Sine-Gordon model is used as a concrete example where these questions about quantum integrability find a complete and affirmative answer. Indeed, in section 3, we show that the SOV characterization of the transfer matrix spectrum is exactly equivalent to a functional equation of the form $\operatorname{det} D(\Lambda)=0$, where $D(\lambda)$ (see (3.21) ) is a one-parameter family of quasi-tridiagonal matrices. In section 4 , we show that this functional equation is indeed equivalent to the Baxter functional equation and, in section 5, we use these results to reconstruct the Baxter Q-operator with the same level of accuracy obtained by the direct construction presented in [1]. It is worth pointing out that these results allow us to prove that the transfer matrix $\mathrm{T}(\lambda)$ (plus the $\Theta$-charge for even chain) describes the family $\mathcal{Q}$ of complete commuting self-adjoint charges which implies the quantum integrability of the model according to definition (1.1). So that in the Sine-Gordon model the Baxter Q-operator plays only the role of a useful auxiliary object.

Let us point out that one of the main advantages of the spectrum characterization derived for the Sine-Gordon model is the possibility to prove an exact reformulation in terms of non-linear integral

\footnotetext{
${ }^{4}$ The Sine-Gordon model at irrational values of the coupling $\beta^{2}$ is a simple case where this kind of problem emerges.

${ }^{5}$ The number of equations in the system is finite and related to the dimension of the cyclic representation.
} 
equation 6 (NLIE). This will be the subject of a future publication where the NLIE characterization will lead us by the implementation of the continuum limit to the description of the Sine-Gordon spectrum in all the interesting regimes. These results will be shown to be consistent with those obtained previously in the literature 7 [37, 38, 39, 40, 41, 42] (see [43, 44] for reviews). Note that the method based on the reformulation of the spectral problem in terms of NLIE has been also used recently [49] to derive the Sinh-Gordon spectrum in finite volume and to characterize the spectrum in the infrared and ultraviolet limits.

The analysis of the Sine-Gordon model allows us to infer the main features required to extend this kind of spectrum characterization to cyclic representations of other integrable quantum models. This is particularly relevant for those models for which a direct construction of the Baxter Q-operator encounters technical difficulties.

Acknowledgments. I would like to thank J. Teschner for stimulating discussions and suggestions on a preliminary version of this work and J.-M. Maillet for the interest shown.

I gratefully acknowledge support from the EC by the Marie Curie Excellence Grant MEXT-CT-2006-042695.

\section{The Sine-Gordon model}

We use this section to recall the main results derived in [1] on the description in terms of SOV of the lattice Sine-Gordon model. This will be used as the starting point to introduce a characterization of the spectrum of the transfer matrix $\mathrm{T}(\lambda)$ which will lead to the construction of the Q-operator from SOV.

\subsection{Definitions}

The lattice Sine-Gordon model can be characterized by the following Lax matrix 8 :

$$
L_{n}^{\mathrm{SG}}(\lambda)=\frac{\kappa_{n}}{i}\left(\begin{array}{cc}
i \mathrm{u}_{n}\left(q^{-\frac{1}{2}} \kappa_{n} \mathrm{v}_{n}+q^{+\frac{1}{2}} \kappa_{n}^{-1} \mathrm{v}_{n}^{-1}\right) & \lambda_{n} \mathrm{v}_{n}-\lambda_{n}^{-1} \mathrm{v}_{n}^{-1} \\
\lambda_{n} \mathrm{v}_{n}^{-1}-\lambda_{n}^{-1} \mathrm{v}_{n} & i \mathrm{u}_{n}^{-1}\left(q^{+\frac{1}{2}} \kappa_{n}^{-1} \mathrm{v}_{n}+q^{-\frac{1}{2}} \kappa_{n} \mathrm{v}_{n}^{-1}\right)
\end{array}\right)
$$

where $\lambda_{n} \equiv \lambda / \xi_{n}$ for any $n \in\{1, \ldots, \mathrm{N}\}$ with $\xi_{n}$ and $\kappa_{n}$ parameters of the model. For any $n \in$ $\{1, \ldots, N\}$ the couple of operators $\left(\mathrm{u}_{n}, \mathrm{v}_{n}\right)$ define a Weyl algebra $\mathcal{W}_{n}$ :

$$
\mathrm{u}_{n} \mathbf{v}_{m}=q^{\delta_{n m}} \mathbf{v}_{m} \mathbf{u}_{n}, \quad \text { where } q=e^{-\pi i \beta^{2}} \text {. }
$$

We will restrict our attention to the case in which $q$ is a root of unity,

$$
\beta^{2}=\frac{p^{\prime}}{p}, \quad p, p^{\prime} \in \mathbb{Z}^{>0},
$$

\footnotetext{
${ }^{6}$ This type of equations were before introduced in a different framework in [35 36

${ }^{7}$ See [45, 46] for a related model analyzed in the framework of ABA and [47, 48] for the corresponding finite volume continuum limit.

${ }^{8}$ The lattice regularization of the Sine-Gordon model that we consider here goes back to [4] [50] and is related to formulations which have more recently been studied in [51 52 53].
} 
with $p \equiv 2 l+1$ odd and $p^{\prime}$ even so that $q^{p}=1$. In this case each Weyl algebra $\mathcal{W}_{n}$ admits a finite-dimensional representation of dimension $p$. In fact, we can represent the operators $\mathrm{u}_{n}, \mathrm{v}_{n}$ on the space of complex-valued functions $\psi: \mathbb{S}_{p}^{N} \rightarrow \mathbb{C}$ as

$$
\begin{aligned}
& \mathrm{u}_{n} \cdot \psi\left(z_{1}, \ldots, z_{\mathrm{N}}\right)=u_{n} z_{n} \psi\left(z_{1}, \ldots, z_{n}, \ldots, z_{\mathrm{N}}\right) \\
& \mathrm{v}_{n} \cdot \psi\left(z_{1}, \ldots, z_{\mathrm{N}}\right)=v_{n} \psi\left(z_{1}, \ldots, q^{-1} z_{n}, \ldots, z_{\mathrm{N}}\right) .
\end{aligned}
$$

where $\mathbb{S}_{p}=\left\{q^{2 n} ; n=0, \ldots, 2 l\right\}$ is a subset of the unit circle; note that $\mathbb{S}_{p}=\left\{q^{n} ; n=0, \ldots, 2 l\right\}$ since $q^{2 l+2}=q$.

The monodromy matrix $\mathrm{M}(\lambda)$ defined in (1.2) in terms of the Lax-matrix (2.5) satisfies the quadratic relations:

$$
R(\lambda / \mu)(\mathrm{M}(\lambda) \otimes 1)(1 \otimes \mathrm{M}(\mu))=(1 \otimes \mathrm{M}(\mu))(\mathrm{M}(\lambda) \otimes 1) R(\lambda / \mu),
$$

where the auxiliary $R$-matrix is given by

$$
R(\lambda)=\left(\begin{array}{cccc}
q \lambda-q^{-1} \lambda^{-1} & & & \\
& \lambda-\lambda^{-1} & q-q^{-1} & \\
& q-q^{-1} & \lambda-\lambda^{-1} & \\
& & & q \lambda-q^{-1} \lambda^{-1}
\end{array}\right)
$$

The elements of $\mathrm{M}(\lambda)$ generate a representation $\mathcal{R}_{\mathrm{N}}$ of the so-called Yang-Baxter algebra characterized by the $4 \mathrm{~N}$ parameters $\kappa=\left(\kappa_{1}, \ldots, \kappa_{\mathrm{N}}\right), \xi=\left(\xi_{1}, \ldots, \xi_{\mathrm{N}}\right), u=\left(u_{1}, \ldots, u_{\mathrm{N}}\right)$ and $v=\left(v_{1}, \ldots, v_{\mathrm{N}}\right)$; in the present paper we will restrict to the case $u_{n}=1, v_{n}=1, n=1, \ldots, \mathrm{N}$. The commutation relations (2.9) are at the basis of the proof of the mutual commutativity of the T-operators.

In the case of a lattice with $\mathrm{N}$ even quantum sites, we have also to introduce the operator:

$$
\Theta=\prod_{n=1}^{\mathrm{N}} \mathrm{v}_{n}^{(-1)^{1+n}}
$$

which plays the role of a grading operator in the Yang-Baxter algebra:

Proposition 6 of [1] $\Theta$ commutes with the transfer matrix and satisfies the following commutation relations with the entries of the monodromy matrix:

$$
\begin{array}{ll}
\Theta \mathrm{C}(\lambda)=q \mathrm{C}(\lambda) \Theta, & {[\mathrm{A}(\lambda), \Theta]=0,} \\
\mathrm{~B}(\lambda) \Theta=q \Theta \mathrm{B}(\lambda), & {[\mathrm{D}(\lambda), \Theta]=0 .}
\end{array}
$$

Moreover, the $\Theta$-charge allows to express the asymptotics of the transfer matrix as:

$$
\lim _{\log \lambda \rightarrow \mp \infty} \lambda^{ \pm \mathrm{N}} \mathrm{T}(\lambda)=\left(\prod_{a=1}^{\mathrm{N}} \frac{\kappa_{a} \xi_{a}^{ \pm 1}}{i}\right)\left(\Theta+\Theta^{-1}\right) .
$$


Let us denote with $\Sigma_{\mathrm{T}}$ the spectrum (the set of the eigenvalue functions $t(\lambda)$ ) of the transfer matrix $\mathrm{T}(\lambda)$. By the definitions (1.2) and (2.5), then $\Sigma_{\mathrm{T}}$ is contained 9 in $\mathbb{C}\left[\lambda^{2}, \lambda^{-2}\right]_{\left(\mathrm{N}+\mathrm{e}_{\mathrm{N}}-1\right) / 2}$, where we have used the notation $\mathrm{e}_{\mathrm{N}}=0$ for $\mathrm{N}$ odd and 1 for $\mathrm{N}$ even.

Note that in the case of $\mathrm{N}$ even, the $\Theta$-charge naturally induces the grading $\Sigma_{\mathrm{T}}=\bigcup_{k=0}^{l} \Sigma_{\mathrm{T}}^{k}$, where:

$$
\Sigma_{\mathbf{\top}}^{k} \equiv\left\{t(\lambda) \in \Sigma_{\mathbf{T}}: \lim _{\log \lambda \rightarrow \mp \infty} \lambda^{ \pm \mathrm{N}} t(\lambda)=\left(\prod_{a=1}^{\mathrm{N}} \frac{\kappa_{a} \xi_{a}^{ \pm 1}}{i}\right)\left(q^{k}+q^{-k}\right)\right\} .
$$

This simply follows by the asymptotics of $\mathrm{T}(\lambda)$ and by its commutativity with $\Theta$. In particular, any $t(\lambda) \in \Sigma_{\boldsymbol{\top}}^{k}$ is a T-eigenvalue corresponding to simultaneous eigenstates of $\mathrm{T}(\lambda)$ and $\Theta$ with $\Theta$-eigenvalues $q^{ \pm k}$.

\subsection{Cyclic SOV representations}

The separation of variables method of Sklyanin is based on the observation that the spectral problem for $\mathrm{T}(\lambda)$ simplifies considerably if one works in an auxiliary representation where the commutative family of operators $B(\lambda)$ is diagonal.

In the case of the Sine-Gordon model the vector space $10 \mathbb{C}^{p^{\mathrm{N}}}$ underlying the SOV representation can be identified with the space of functions $\Psi(\eta)$ defined for $\eta$ taken from the discrete set

$$
\mathbb{B}_{\mathrm{N}} \equiv\left\{\left(q^{k_{1}} \zeta_{1}, \ldots, q^{k_{\mathrm{N}}} \zeta_{\mathrm{N}}\right) ;\left(k_{1}, \ldots, k_{\mathrm{N}}\right) \in \mathbb{Z}_{p}^{\mathrm{N}}\right\}
$$

on these functions $\mathrm{B}(\lambda)$ acts as a multiplication operator,

$$
\mathrm{B}_{\mathrm{N}}(\lambda) \Psi(\eta)=\eta_{\mathrm{N}}^{\mathrm{e}_{\mathrm{N}}} b_{\eta}(\lambda) \Psi(\eta), \quad b_{\eta}(\lambda) \equiv \prod_{n=1}^{\mathrm{N}} \frac{\kappa_{n}}{i} \prod_{a=1}^{[\mathrm{N}]}\left(\lambda / \eta_{a}-\eta_{a} / \lambda\right)
$$

where $[\mathrm{N}] \equiv \mathrm{N}-\mathrm{e}_{\mathrm{N}}$ and $\eta_{1}, \ldots, \eta_{[\mathrm{N}]}$ are the zeros of $b_{\eta}(\lambda)$. In the case of even $\mathrm{N}$ it turns out that we need a supplementary variable $\eta_{\mathrm{N}}$ in order to be able to parameterize the spectrum of $\mathrm{B}(\lambda)$.

In [1] we have proven that for general values of the parameters $\kappa$ and $\xi$ of the original representation it is possible to construct these SOV representations and moreover we have defined the map which fixes the SOV parameter $\eta$ in terms of the parameters $\kappa$ and $\xi$.

In these SOV representations the spectral problem for $T(\lambda)$ is reduced to the following discrete system of Baxter-like equations in the wave-function $\Psi_{t}(\eta)=\langle\eta \mid t\rangle$ of a T-eigenstate $|t\rangle$ :

$$
t\left(\eta_{r}\right) \Psi(\eta)=\mathrm{a}\left(\eta_{r}\right) \mathrm{T}_{r}^{-} \Psi(\eta)+\mathrm{d}\left(\eta_{r}\right) \mathrm{T}_{r}^{+} \Psi(\eta) \quad \forall r \in\{1, \ldots,[\mathrm{N}]\},
$$

\footnotetext{
${ }^{9}$ Here with $\mathbb{C}\left[x, x^{-1}\right]_{M}$ we are denoting the linear space of the Laurent polynomials of degree $M$ in the variable $x \in \mathbb{C}$.

${ }^{10}$ It is always possible to provide the structure of Hilbert space to this finite-dimensional linear space. In particular, the scalar product in the SOV space is naturally introduced by the requirement that the transfer matrix is self-adjoint in the SOV representation. Appendix B addresses this issue.
} 
where $\mathrm{T}_{r}^{ \pm}$are the operators defined by

$$
\mathrm{T}_{r}^{ \pm} \Psi\left(\eta_{1}, \ldots, \eta_{\mathrm{N}}\right)=\Psi\left(\eta_{1}, \ldots, q^{ \pm 1} \eta_{r}, \ldots, \eta_{\mathrm{N}}\right),
$$

while the coefficients $\mathrm{a}(\lambda)$ and $\mathrm{d}(\lambda)$ are defined by:

$$
\mathrm{a}(\lambda)=\prod_{n=1}^{\mathrm{N}} \frac{\kappa_{n}}{i \lambda_{n}}\left(1-i q^{-1 / 2} \lambda_{n} \kappa_{n}\right)\left(1-i q^{-1 / 2} \frac{\lambda_{n}}{\kappa_{n}}\right), \quad \mathrm{d}(\lambda)=q^{\mathrm{N}} \mathrm{a}(-\lambda q) .
$$

In the case of $\mathrm{N}$ even we have to add to the system (2.18) the following equation in the variable $\eta_{\mathrm{N}}$ :

$$
\mathrm{T}_{\mathrm{N}}^{+} \Psi_{ \pm k}(\eta)=q^{ \pm k} \Psi_{ \pm k}(\eta),
$$

for $t(\lambda) \in \Sigma_{\mathrm{T}}^{k}$ with $k \in\{0, \ldots, l\}$. Note that the cyclicity of these SOV representations is expressed by the identification of $\left(\mathrm{T}_{j}^{ \pm}\right)^{p}$ with the identity for any $j \in\{1, \ldots, \mathrm{N}\}$.

\section{SOV characterization of T-eigenvalues}

Let us introduce the one parameter family $D(\lambda)$ of $p \times p$ matrix:

$$
D(\lambda) \equiv\left(\begin{array}{cccccc}
t(\lambda) & -\mathrm{d}(\lambda) & 0 & \ldots & 0 & -\mathrm{a}(\lambda) \\
-\mathrm{a}(q \lambda) & t(q \lambda) & -\mathrm{d}(q \lambda) & 0 & \cdots & 0 \\
0 & \ddots & & & & \vdots \\
\vdots & & \ldots & & & \vdots \\
\vdots & & & \ldots & & \vdots \\
\vdots & & & & \ddots & 0 \\
0 & \ldots & 0 & -\mathrm{a}\left(q^{2 l-1} \lambda\right) & t\left(q^{2 l-1} \lambda\right) & -\mathrm{d}\left(q^{2 l-1} \lambda\right) \\
-\mathrm{d}\left(q^{2 l} \lambda\right) & 0 & \ldots & 0 & -\mathrm{a}\left(q^{2 l} \lambda\right) & t\left(q^{2 l} \lambda\right)
\end{array}\right)
$$

where for now $t(\lambda)$ is just an even Laurent polynomial of degree $\mathrm{N}+\mathrm{e}_{\mathrm{N}}-1$ in $\lambda$.

Lemma 1. The determinant $\operatorname{det}_{p} D$ is an even Laurent polynomial of maximal degree $\mathrm{N}+e_{\mathrm{N}}-1$ in $\Lambda \equiv \lambda^{p}$.

Proof. Let us start observing that $D(\lambda q)$ is obtained by $D(\lambda)$ exchanging the first and $p$-th column and after the first and $p$-th row, so that

$$
\operatorname{det}_{p} D(\lambda q)=\operatorname{det}_{p} D(\lambda) \quad \forall \lambda \in \mathbb{C},
$$

which implies that $\operatorname{det}_{p} D$ is function of $\Lambda$. Let us develop the determinant:

$$
\begin{aligned}
\operatorname{det}_{p} D(\Lambda)= & \prod_{h=1}^{p} \mathrm{a}\left(\lambda q^{h}\right)+\prod_{h=1}^{p} \mathrm{a}\left(-\lambda q^{h}\right)-q^{\mathrm{N}} \mathrm{a}(\lambda) \mathrm{a}(-\lambda) \operatorname{det}_{2 l-1} D_{(1,2 l+1),(1,2 l+1)}(\lambda) \\
& -q^{\mathrm{N}} \mathrm{a}(\lambda q) \mathrm{a}(-\lambda q) \operatorname{det}_{2 l-1} D_{(1,2),(1,2)}(\lambda)+t(\lambda) \operatorname{det}_{2 l} D_{1,1}(\lambda),
\end{aligned}
$$


where $D_{(h, k),(h, k)}(\lambda)$ denotes the $(2 l-1) \times(2 l-1)$ sub-matrix of $D(\lambda)$ obtained removing the rows and columns $h$ and $k$ while $D_{h, k}(\lambda)$ denotes the $2 l \times 2 l$ sub-matrix of $D(\lambda)$ obtained removing the row $h$ and column $k$. The interest toward this decomposition of $\operatorname{det}_{p} D(\Lambda)$ is due to the fact that the matrices $D_{(1,2),(1,2)}(\lambda), D_{(1,2 l+1),(1,2 l+1)}(\lambda)$ and $D_{1,1}(\lambda)$ are tridiagonal matrices. Following the same reasoning used in Lemma4 to prove that $\operatorname{det}_{2 l} D_{1,1}(\lambda)$ is an even function of $\lambda$ we can also show that this is true for $\operatorname{det}_{2 l-1} D_{(1,2),(1,2)}(\lambda)$ and $\operatorname{det}_{2 l-1} D_{(1,2 l+1),(1,2 l+1)}(\lambda)$. From the parity of these functions the parity of $\operatorname{det}_{p} D(\Lambda)$ follows by using $(3.23)$.

Being a $(\lambda), \mathrm{d}(\lambda)$ and $t(\lambda)$ Laurent polynomial of degree $\mathrm{N}$ in $\lambda$, in the case of $\mathrm{N}$ even the statement of the lemma is already proven; so we have just to show that:

$$
\lim _{\log \Lambda \rightarrow \mp \infty} \Lambda^{ \pm \mathrm{N}} \operatorname{det}_{p} D(\Lambda)=0
$$

for $\mathrm{N}$ odd which follows observing that:

$$
\lim _{\log \Lambda \rightarrow \mp \infty} \Lambda^{ \pm \mathrm{N}} \operatorname{det}_{p} D(\Lambda)=i^{ \pm p N} \prod_{n=1}^{\mathrm{N}} \kappa_{n}^{p} \xi_{n}^{ \pm p} \operatorname{det}_{p}\left\|q^{-(1 \mp 1) \mathrm{N} / 2} \delta_{h, k+1}-q^{(1 \mp 1) \mathrm{N} / 2} \delta_{h, k-1}\right\| .
$$

The interest toward the function $\operatorname{det}_{p} D(\Lambda)$ is due to the fact that it allows the following characterization of the T-spectrum:

Lemma 2. $\Sigma_{\mathrm{T}}$ is the set of all the functions $t(\lambda) \in \mathbb{C}\left[\lambda^{2}, \lambda^{-2}\right]_{\left(\mathrm{N}+e_{\mathrm{N}}-1\right) / 2}$ which satisfy the system of equations:

$$
\underset{p}{\operatorname{det}} D\left(\eta_{a}^{p}\right)=0 \quad \forall a \in\{1, \ldots,[\mathrm{N}]\} \text { and }\left(\eta_{1}, \ldots, \eta_{[\mathrm{N}]}\right) \in \mathbb{B}_{\mathrm{N}},
$$

plus in the case of $\mathrm{N}$ even:

$$
\lim _{\log \Lambda \rightarrow \infty} \Lambda^{ \pm \mathrm{N}} \underset{p}{\operatorname{det}} D(\Lambda)=0 .
$$

Proof. The requirement that the system of equations (2.18) admits a non-zero solution leads to the equations (3.26), while the equation (3.27) for even $\mathrm{N}$ simply follows by observing that:

$$
\begin{aligned}
\lim _{\log \Lambda \rightarrow \mp \infty} \Lambda_{p}^{ \pm \mathrm{N}} \operatorname{det}_{p} D(\Lambda) & =\operatorname{det}_{p}\left\|q^{(1 \mp 1) \mathrm{N} / 2} \delta_{i, j-1}+q^{-(1 \mp 1) \mathrm{N} / 2} \delta_{i, j+1}-\left(q^{k}+q^{-k}\right) \delta_{i, j}\right\| \\
& \times(-1) \prod_{n=1}^{\mathrm{N}}\left(i \kappa_{n} \xi_{n}^{ \pm}\right)^{p}=0
\end{aligned}
$$

Note that the above characterization of the T-spectrum $\Sigma_{\mathrm{T}}$ requires as input the knowledge of $\mathbb{B}_{\mathrm{N}}$, i.e. the lattice of zeros of the operator $B(\lambda)$. It is so interesting to notice that this characterization 
has in fact a reformulation which is independent from the knowledge of $\mathbb{B}_{N}$. To explain this let us note that Lemma 1 allows to introduce the following map:

$$
\mathcal{D}_{p, \mathrm{~N}}: t(\lambda) \in \mathbb{C}\left[\lambda^{2}, \lambda^{-2}\right]_{\left(\mathrm{N}+\mathrm{e}_{\mathrm{N}}-1\right) / 2} \rightarrow \mathcal{D}_{p, \mathrm{~N}}(t(\lambda)) \equiv \operatorname{det}_{p} D(\Lambda) \in \mathbb{C}\left[\Lambda^{2}, \Lambda^{-2}\right]_{\left(\mathrm{N}+\mathrm{e}_{\mathrm{N}}-1\right) / 2} .
$$

In terms of this map we can introduce a further characterization of the spectrum of the transfer matrix $\mathrm{T}(\lambda)$.

Theorem 1. The spectrum $\Sigma_{\mathrm{T}}$ of the transfer matrix $\mathrm{T}(\lambda)$ coincides with the kernel $\mathcal{N}_{\mathcal{D}_{p, \mathrm{~N}}} \subset$ $\mathbb{C}\left[\lambda^{2}, \lambda^{-2}\right]_{\left(\mathrm{N}+e_{\mathrm{N}}-1\right) / 2}$ of the map $\mathcal{D}_{p, \mathrm{~N}}$.

Proof. The inclusion $\mathcal{N}_{\mathcal{D}_{p, \mathrm{~N}}} \subset \Sigma_{\mathrm{T}}$ is trivial thanks to Lemma 2, vice-versa if $t(\lambda) \in \Sigma_{\mathrm{T}}$ then the function $\operatorname{det}_{p} D(\Lambda)$ is zero in $\mathrm{N}+\mathrm{e}_{\mathrm{N}}$ different values of $\Lambda^{2}$ which thanks to Lemma 1 implies $\operatorname{det}_{p} D(\Lambda) \equiv 0$, i.e. $\Sigma_{\mathrm{T}} \subset \mathcal{N}_{\mathcal{D}_{p, \mathrm{~N}}}$.

That is the set of eigenvalues of the transfer matrix $\mathrm{T}(\lambda)$ is exactly characterized as the subset of $\mathbb{C}\left[\lambda^{2}, \lambda^{-2}\right]_{\left(\mathrm{N}+\mathrm{e}_{\mathrm{N}}-1\right) / 2}$ which contains all the solutions of the functional equation $\operatorname{det}_{p} D(\Lambda)=0$. In the next section we will show that this functional equation is nothing else that the Baxter equation.

Remark 1. Let us note that the same kind of functional equation $\operatorname{det} D(\Lambda)=0$ also appears in [54, 55, 56]. There it recasts, in a compact form, the functional relations which result from the truncated fusions of transfer matrix eigenvalues. It is so relevant to point out that for the BBS-mode 11 in the SOV representation the non-triviality condition of the solutions of the system of Baxter-like equations has been shown [60] to be equivalent to the truncation identity in the fusion of transfer matrix eigenvalues.

\section{Baxter functional equation}

The main consequence of the previous analysis is that it naturally leads to the complete characterization of the transfer matrix spectrum in terms of polynomial solutions of the Baxter functional equation.

Theorem 2. Let $t(\lambda) \in \Sigma_{\mathrm{T}}$ then $t(\lambda)$ defines uniquely up to normalization a polynomial $Q_{t}(\lambda)$ that satisfies the Baxter functional equation:

$$
t(\lambda) Q_{t}(\lambda)=\mathrm{a}(\lambda) Q_{t}\left(\lambda q^{-1}\right)+\mathrm{d}(\lambda) Q_{t}(\lambda q) \quad \forall \lambda \in \mathbb{C} .
$$

Proof. The fact that given a $t(\lambda) \in \mathbb{C}\left[\lambda^{2}, \lambda^{-2}\right]_{\left(\mathrm{N}+\mathrm{e}_{\mathrm{N}}-1\right) / 2}$ there exists up to normalization at most one polynomial $Q_{t}(\lambda)$ that satisfies the Baxter functional equation has been proven in Lemma 2 of [1]. So we have to prove only the existence of $Q_{t}(\lambda) \in \mathbb{C}[\lambda]$. An interesting point about the proof given here is that it is a constructive proof.

\footnotetext{
${ }^{11}$ The BBS-model [12 $\left[\begin{array}{l|l|l|}57 & 58 & 59]\end{array}\right.$ has been analyzed in the SOV approach in a series of works [60 61 62].
} 
Let us notice that the condition $t(\lambda) \in \Sigma_{\mathbf{T}} \equiv \mathcal{N}_{\mathcal{D}_{p, \mathrm{~N}}}$ implies that the $p \times p$ matrix $D(\lambda)$ has rank $2 l$ for any $\lambda \in \mathbb{C} \backslash\{0\}$. Let us denote with

$$
\mathrm{C}_{i, j}(\lambda)=(-1)^{i+j} \operatorname{det}_{2 l} D_{i, j}(\lambda)
$$

the $(i, j)$ cofactor of the matrix $D(\lambda)$; then the matrix formed out of these cofactors has rank 1, i.e. all the vectors:

$$
\mathrm{V}_{i}(\lambda) \equiv\left(\mathrm{C}_{i, 1}(\lambda), \mathrm{C}_{i, 2}(\lambda), \ldots, \mathbf{C}_{i, 2 l+1}(\lambda)\right)^{\top} \in \mathbb{C}^{p} \quad \forall i \in\{1, \ldots, 2 l+1\}
$$

are proportional:

$$
\mathrm{V}_{i}(\lambda) / \mathrm{C}_{i, 1}(\lambda)=\mathrm{V}_{j}(\lambda) / \mathrm{C}_{j, 1}(\lambda) \quad \forall i, j \in\{1, \ldots, 2 l+1\}, \forall \lambda \in \mathbb{C} .
$$

The proportionality (4.33) of the eigenvectors $\mathrm{V}_{i}(\lambda)$ implies:

$$
\mathrm{C}_{2,2}(\lambda) / \mathrm{C}_{2,1}(\lambda)=\mathrm{C}_{1,2}(\lambda) / \mathrm{C}_{1,1}(\lambda)
$$

which, by using the property A.69), can be rewritten as:

$$
\mathrm{C}_{1,1}(\lambda q) / \mathrm{C}_{1,2 l+1}(\lambda q)=\mathrm{C}_{1,2}(\lambda) / \mathrm{C}_{1,1}(\lambda) .
$$

Moreover, the first element in the vectorial condition $D(\lambda) \mathrm{V}_{1}(\lambda)=\underline{0}$ reads:

$$
t(\lambda) \mathrm{C}_{1,1}(\lambda)=\mathrm{a}(\lambda) \mathrm{C}_{1,2 l+1}(\lambda)+\mathrm{d}(\lambda) \mathrm{C}_{1,2}(\lambda) .
$$

Let us note that from the form of $\mathrm{a}(\lambda), \mathrm{d}(\lambda)$ and $t(\lambda) \in \Sigma_{\mathrm{T}}$ it follows that all the cofactors are Laurent polynomial of maximal degree $122 l \mathrm{~N}$ in $\lambda$ :

$$
\mathrm{C}_{i, j}(\lambda)=\mathrm{C}_{i, j} \lambda^{-2 l \mathrm{~N}+a_{i, j}} \prod_{h=1}^{4 l \mathrm{~N}-\left(a_{i, j}+b_{i, j}\right)}\left(\lambda_{h}^{(i, j)}-\lambda\right) .
$$

In Lemma 5 we show that the equations (4.35) and (4.36) imply that if $\mathrm{C}_{1,1}(\lambda)$ has a common zero with $\mathrm{C}_{1,2}(\lambda)$ then this is also a zero of $\mathrm{C}_{1,2 l+1}(\lambda)$ and that the same statement holds exchanging $\mathrm{C}_{1,2}(\lambda)$ with $\mathrm{C}_{1,2 l+1}(\lambda)$. So we can denote with $\mathrm{C}_{1,1} \overline{\mathrm{C}}_{1,1}(\lambda), \mathrm{C}_{1,2 l+1} \overline{\mathrm{C}}_{1,2 l+1}(\lambda)$ and $\mathrm{C}_{1,2} \overline{\mathrm{C}}_{1,2}(\lambda)$ the polynomials of maximal degree $4 \mathrm{lN}$ obtained simplifying the common factors in $\mathrm{C}_{1,1}(\lambda), \mathrm{C}_{1,2 l+1}(\lambda)$ and $\mathrm{C}_{1,2}(\lambda)$. Then, by equation (4.35), they have to satisfy the relations:

$$
\overline{\mathrm{C}}_{1,2 l+1}(\lambda)=q^{\overline{\mathrm{N}}_{1,1}} \overline{\mathrm{C}}_{1,1}\left(\lambda q^{-1}\right), \quad \overline{\mathrm{C}}_{1,2}(\lambda)=q^{-\overline{\mathrm{N}}_{1,1}} \overline{\mathrm{C}}_{1,1}(\lambda q) \text { and } \mathrm{C}_{1,2 l+1}=\varphi \mathrm{C}_{1,1},
$$

where $\varphi \equiv \mathrm{C}_{1,1} / \mathrm{C}_{1,2}$ and $\overline{\mathrm{N}}_{1,1}$ is the degree of the polynomial $\overline{\mathrm{C}}_{1,1}(\lambda)$. So that equation (4.36) assumes the form of a Baxter equation in the polynomial $\overline{\mathrm{C}}_{1,1}(\lambda)$ :

$$
t(\lambda) \overline{\mathrm{C}}_{1,1}(\lambda)=\overline{\mathrm{a}}(\lambda) \overline{\mathrm{C}}_{1,1}\left(\lambda q^{-1}\right)+\overline{\mathrm{d}}(\lambda) \overline{\mathrm{C}}_{1,1}(\lambda q),
$$

\footnotetext{
${ }^{12}$ The $a_{i, j}$ and $b_{i, j}$ are non-negative integers and $\lambda_{h}^{(i, j)} \neq 0$ for any $h \in\left\{1, \ldots, 4 l \mathrm{~N}-\left(a_{i, j}+b_{i, j}\right)\right\}$.
} 
with coefficients $\overline{\mathrm{a}}(\lambda) \equiv q^{\overline{\mathrm{N}}_{1,1}} \varphi \mathrm{a}(\lambda)$ and $\overline{\mathrm{d}}(\lambda) \equiv q^{-\overline{\mathrm{N}}_{1,1}} \varphi^{-1} \mathrm{~d}(\lambda)$. Note that the consistence of the above equation implies that $\varphi$ is a $p$-root of the unity. Indeed, denoting with $\bar{D}(\Lambda)$ the matrix defined as in (3.21) but with coefficients $\overline{\mathrm{a}}(\lambda)$ and $\overline{\mathrm{d}}(\lambda)$, equation (4.39) implies:

$$
0=\operatorname{det}_{p} \bar{D}(\Lambda) \equiv\left(\varphi^{p}-1\right)\left(\prod_{h=1}^{p} \mathrm{a}\left(\lambda q^{h}\right)-\varphi^{-p} \prod_{h=1}^{p} \mathrm{a}\left(-\lambda q^{h}\right)\right) .
$$

The expansion for $\operatorname{det}_{p} \bar{D}(\Lambda)$ in (4.40) is derived by using the expansion (3.23) for $\operatorname{det}_{p} \bar{D}(\Lambda)$, the formulae13:

$$
\begin{aligned}
& \operatorname{det} \bar{D}_{1,1}(\lambda)=\operatorname{det} D_{1,1}(\lambda), \\
& \operatorname{det}_{2 l-1} \bar{D}_{(1,2),(1,2)}(\lambda)=\operatorname{det}_{2 l-1} D_{(1,2),(1,2)}(\lambda), \\
& \operatorname{det} \bar{D}_{(1,2 l+1),(1,2 l+1)}(\lambda)=\operatorname{det}_{2 l-1} D_{(1,2 l+1),(1,2 l+1)}(\lambda),
\end{aligned}
$$

and the condition $t(\lambda) \in \Sigma_{T}$. Finally, if we define:

$$
Q_{t}(\lambda) \equiv \lambda^{a} \overline{\mathbf{C}}_{1,1}(\lambda)
$$

where $q^{-a}=q^{\overline{\mathrm{N}}_{1,1}} \varphi$ with $a \in\{0, . ., 2 l\}$, we get the statement of the theorem.

Remark 2. The previous theorem implies that for any $t(\lambda) \in \Sigma_{\boldsymbol{T}}$ the polynomial solution $Q_{t}(\lambda)$ of the Baxter equation can be related to the determinant of a tridiagonal matrix of finite size $p-1$. Note that the spectrum of the Sine-Gordon model in the case of irrational coupling $\bar{\beta}^{2}$ should be deduced from $\beta^{2}=p^{\prime} / p$ rational in the limit $\beta^{2} \rightarrow \bar{\beta}^{2}$. In particular, this implies that under this limit $(p \rightarrow$ $+\infty$ ) the dimension of the representation diverges as well as the size of the tridiagonal matrix whose determinant is associated to the solution $Q_{t}(\lambda)$ of the Baxter equation. It is then relevant to point out that in the case of the quantum periodic Toda chain the solutions of the corresponding Baxter equation are expressed in terms of determinants of semi-infinite tridiagonal matrices [63, 13, 64].

It is worth noticing that the set of polynomials $Q_{t}(\lambda)$, introduced in the previous theorem, admits a more precise characterization:

Theorem 3. Let $t(\lambda) \in \Sigma_{\mathrm{T}}$ then $t(\lambda)$ defines uniquely up to normalization a polynomial solution $Q_{t}(\lambda)$ of the Baxter functional equation (4.30) of maximal degree $2 l \mathrm{~N}$.

In the case $\mathrm{N}$ odd, it results:

$$
Q_{t}(0) \equiv Q_{0} \neq 0, \text { and } \lim _{\lambda \rightarrow \infty} \lambda^{-2 l \mathrm{~N}} Q_{t}(\lambda) \equiv Q_{2 l \mathrm{~N}} \neq 0 .
$$

In the case $\mathrm{N}$ even, the condition (4.45) selects $t(\lambda) \in \Sigma_{\mathrm{T}}^{0}$ while for $t(\lambda) \in \Sigma_{\mathrm{T}}^{k}$ with $k \in\{1, \ldots, l\}$ we have the characterization $Q_{0}=Q_{2 l \mathrm{~N}}=0$ and:

$$
\lim _{\lambda \rightarrow 0} \frac{Q_{t}(\lambda q)}{Q_{t}(\lambda)}=q^{ \pm k}, \quad \lim _{\lambda \rightarrow \infty} \frac{Q_{t}(\lambda q)}{Q_{t}(\lambda)}=q^{-(\mathrm{N} \pm k)} .
$$

\footnotetext{
${ }^{13}$ They follow from the tridiagonality of these matrices and by using Lemma 3
} 
Proof. Thanks to formula A.74), the cofactor $\mathrm{C}_{1,1}(\lambda) \in \mathbb{C}\left[\lambda, \lambda^{-1}\right]_{2 l \mathrm{~N}}$ is even in $\lambda$ and so it admits the expansions:

$$
\mathrm{C}_{1,1}(\lambda)=\mathrm{C}_{1,1} \lambda^{-2 l \mathrm{~N}+2 \tilde{a}_{1,1}} \prod_{i=1}^{2 l \mathrm{~N}-\left(\tilde{a}_{1,1}+\tilde{b}_{1,1}\right)}\left(\lambda_{i}^{(1,1)}-\lambda\right)\left(\lambda_{i}^{(1,1)}+\lambda\right) .
$$

Let us note now that by using the properties (A.69) and (A.74), the relation (4.34) can be rewritten as:

$$
\mathrm{C}_{1,1}(\lambda q) \mathbf{C}_{1,1}(\lambda)=q^{\mathrm{N}} \mathbf{C}_{1,2}(\lambda) \mathbf{C}_{1,2}(-\lambda) .
$$

Using that and the general representation (4.37) for the cofactor $\mathrm{C}_{1,2}(\lambda)$, we get:

$$
a_{1,2}=2 \tilde{a}_{1,1} \equiv 2 a, \quad b_{1,2}=2 \tilde{b}_{1,1} \equiv 2 b, \quad \mathrm{C}_{1,2}^{2}=\mathrm{C}_{1,1}^{2} q^{-2(\mathrm{~N}+b)}
$$

and:

$$
\left(\lambda_{i}^{(1,1)}\right)^{2}=\left(\lambda_{i}^{(1,2)}\right)^{2} \equiv \bar{\lambda}_{i}^{2}, \quad\left(\lambda_{i+2 l N-(a+b)}^{(1,2)}\right)^{2}=\left(\bar{\lambda}_{i} / q\right)^{2}
$$

with $\bar{\lambda}_{i} \neq 0$ for any $i \in\{1, \ldots, 2 l \mathrm{~N}-(a+b)\}$ with $a$ and $b \in \mathbb{Z}^{\geq 0}$. Note that the equation (4.49) and the fact that $\varphi \equiv \mathrm{C}_{1,1} / \mathrm{C}_{1,2}$ is a $p$-root of the unity imply $\varphi=q^{b+\mathrm{N}}$. Then we can write:

$$
\begin{aligned}
& \mathrm{C}_{1,1}(\lambda)=\mathrm{C} \lambda^{-2 l \mathrm{~N}+2 a} \prod_{i=1}^{2 l \mathrm{~N}-(a+b)}\left(\bar{\lambda}_{i}+\lambda\right)\left(\bar{\lambda}_{i}-\lambda\right), \\
& \mathrm{C}_{1,2}(\lambda)=q^{a} \mathrm{C} \lambda^{-2 l \mathrm{~N}+2 a} \prod_{i=1}^{2 l \mathrm{~N}-(a+b)}\left(\bar{\lambda}_{i}+\lambda\right)\left((-1)^{H(x-i)} \bar{\lambda}_{i}-\lambda q\right),
\end{aligned}
$$

where $\mathrm{C} \equiv \mathrm{C}_{1,1}$ and $H(n) \equiv\{0$ for $n<0, \quad 1$ for $n \geq 0\}$ is the Heaviside step function. Here, $x$ is a non-negative integer which is fixed to zero thanks to formula (4.38). Then the solution $Q_{t}(\lambda)$ of the Baxter equation (4.30) belongs to $\mathbb{C}[\lambda]_{2 l \mathrm{~N}}$ and has the form:

$$
Q_{t}(\lambda) \equiv \lambda^{a} \prod_{i=1}^{2 l N-(a+b)}\left(\bar{\lambda}_{i}-\lambda\right) .
$$

Let us show now the remaining statements of the theorem concerning the asymptotics of $Q_{t}(\lambda)$. To this aim we compute the limits:

$$
\begin{aligned}
\lim _{\log \lambda \rightarrow \mp \infty} \lambda^{ \pm 2 l \mathrm{~N}} \mathrm{C}_{1,1}(\lambda) & =\operatorname{det}_{2 l}\left\|q^{-(1 \mp 1) \mathrm{N} / 2} \delta_{i, j+1}+q^{(1 \mp 1) \mathrm{N} / 2} \delta_{i, j-1}-\left(q^{k}+q^{-k}\right) \delta_{\mathrm{e}_{\mathrm{N}}, 1} \delta_{i, j}\right\|_{i \neq 1, j \neq 1} \\
& \times \prod_{h=1}^{\mathrm{N}}\left(\frac{\kappa_{h} \xi_{h}^{ \pm 1}}{i}\right)^{2 l}=\left(\delta_{\mathrm{e}_{\mathrm{N}}, 1}\left(1+(2 l+1) \delta_{k, 0}\right)-1\right) \prod_{h=1}^{\mathrm{N}}\left(\frac{\kappa_{h} \xi_{h}^{\mp 1}}{i}\right)^{2 l},
\end{aligned}
$$

which imply:

$$
a=b=0,
$$


for $\mathrm{N}$ odd and $\mathrm{N}$ even with $t(\lambda) \in \Sigma_{\mathrm{T}}^{0}$, i.e. the condition (4.45). In the remaining cases, $\mathrm{N}$ even and $t(\lambda) \notin \Sigma_{\mathrm{T}}^{0}$, the same formula implies:

$$
a \neq 0, b \neq 0,
$$

so that $Q_{0}=Q_{2 l \mathrm{~N}}=0$, while the asymptotics behaviors (4.46) simply follow taking the asymptotics of the Baxter equation satisfied by $Q_{t}(\lambda)$.

\section{Q-operator: Existence and characterization}

Let us denote with $\boldsymbol{\Sigma}_{t}$ the eigenspace of the transfer matrix $\mathrm{T}(\lambda)$ corresponding to the eigenvalue $t(\lambda) \in \Sigma_{\mathrm{T}}$, then:

Definition 1. Let $\mathrm{Q}(\lambda)$ be the operator family defined by:

$$
\mathrm{Q}(\lambda)|t\rangle \equiv Q_{t}(\lambda)|t\rangle \quad \forall|t\rangle \in \boldsymbol{\Sigma}_{t} \text { and } \forall t(\lambda) \in \Sigma_{\mathbf{T}},
$$

with $Q_{t}(\lambda)$ the element of $\mathbb{C}[\lambda]_{2 l \mathrm{~N}}$ corresponding to $t(\lambda) \in \Sigma_{\mathrm{T}}$ by the injection defined in the previous theorem.

Under the assumptions $\xi$ and $\kappa$ real or imaginary numbers, which assure the self-adjointness of the transfer matrix $\mathrm{T}(\lambda)$ for $\lambda \in \mathbb{R}$, the following theorem holds:

Theorem 4. The operator family $\mathrm{Q}(\lambda)$ is a Baxter $\mathrm{Q}$-operator:

(A) $\mathrm{Q}(\lambda)$ satisfies with $\mathrm{T}(\lambda)$ the commutation relations:

$$
[\mathrm{Q}(\lambda), \mathrm{T}(\mu)]=[\mathrm{Q}(\lambda), \mathrm{Q}(\mu)]=0 \quad \forall \lambda, \mu \in \mathbb{C},
$$

plus the Baxter equation:

$$
\mathrm{T}(\lambda) \mathrm{Q}(\lambda)=\mathrm{a}(\lambda) \mathrm{Q}\left(\lambda q^{-1}\right)+\mathrm{d}(\lambda) \mathrm{Q}(\lambda q) \quad \forall \lambda \in \mathbb{C} .
$$

(B) $\mathrm{Q}(\lambda)$ is a polynomial of degree $2 l \mathrm{~N}$ in $\lambda$ :

$$
\mathrm{Q}(\lambda) \equiv \sum_{n=0}^{2 l \mathrm{~N}} \mathrm{Q}_{n} \lambda^{n},
$$

with coefficients $Q_{n}$ self-adjoint operators.

(C) In the case $\mathrm{N}$ odd, the operator $\mathrm{Q}_{2 l \mathrm{~N}}=i d$ and $\mathrm{Q}_{0}$ is an invertible operator.

(D) In the case $\mathrm{N}$ even, $\mathrm{Q}(\lambda)$ commutes with the $\Theta$-charge and the operator $\mathrm{Q}_{2 l \mathrm{~N}}$ is the orthogonal projection onto the $\Theta$-eigenspace with eigenvalue $1 . Q_{0}$ has non-trivial kernel coinciding with the orthogonal complement to the $\Theta$-eigenspace with eigenvalue 1 . 
Proof. Note that the self-adjointness of the transfer matrix $\mathrm{T}(\lambda)$ implies that $\mathrm{Q}(\lambda)$ is well defined, indeed its action is defined on a basis. The property (A) is a trivial consequence of Definition 1. Note that the injectivity of the map $t(\lambda) \in \Sigma_{\mathrm{T}} \rightarrow Q_{t}(\lambda) \in \mathbb{C}[\lambda]_{2 l \mathrm{~N}}$ implies:

$$
\left(Q_{t}(\lambda)\right)^{*}=Q_{t}\left(\lambda^{*}\right) \forall \lambda \in \mathbb{C}
$$

being $(\mathrm{a}(\lambda))^{*}=\mathrm{d}\left(\lambda^{*}\right)$ and $(t(\lambda))^{*}=t\left(\lambda^{*}\right)$. So we get the Hermitian conjugation property $(\mathrm{Q}(\lambda))^{\dagger}=\mathrm{Q}\left(\lambda^{*}\right)$, i.e. the self-adjointness of the operators $\mathrm{Q}_{n}$. The properties (C) and (D) of the operators $Q_{0}$ and $Q_{2 l N}$ directly follow from the asymptotics of the eigenfunction $Q_{t}(\lambda)$ while the commutativity of $\mathrm{Q}(\lambda)$ and $\Theta$ is a direct consequence of the commutativity of $\mathrm{T}(\lambda)$ and $\Theta$.

\section{Conclusion}

In the previous section we have shown that by only using the characterization of the spectrum of the transfer matrix obtained by the SOV method we were able to reconstruct the Q-operator. It is also interesting to point out as the results derived in [1] together with those of the present article yield:

Theorem 5. The family $\mathcal{Q}$ which characterizes the quantum integrability of the lattice Sine-Gordon model (see definition (1.1) is described by the transfer matrix $\mathrm{T}(\lambda)$ for a chain with $\mathrm{N}$ odd number of sites while by $\mathrm{T}(\lambda)$ plus the $\Theta$-charge for a chain with $\mathrm{N}$ even number of sites.

Proof. Let us start noticing that Proposition 3 and Theorem 4 of [1] are derived only using the SOV method (i.e. without any assumption about the existence of the Q-operator). So only using SOV analysis we have derived that for $\mathrm{N}$ odd the transfer matrix $\mathrm{T}(\lambda)$ has simple spectrum while for $\mathrm{N}$ even this is true for $\mathrm{T}(\lambda)$ plus the $\Theta$-charge; i.e. they define a complete family of commuting observables and so satisfy the properties (A) and (C) of the definition (1.1). In this article we have moreover shown that the $\mathrm{Q}$-operator is defined as a function of the transfer matrix which implies the property (B) of (1.1) recalling that in [1] the time-evolution operator $\mathrm{U}$ has been expressed as a function of the $\mathrm{Q}$-operator.

Let us shortly point out the main features required in abstract to extend to cyclic representations of other integrable quantum models the same kind of spectrum characterization derived here for the lattice Sine-Gordon model.

R1. The model admits an SOV description and the spectrum of the transfer matrix can be characterized by a system of Baxter-like equations in the T-wave-function $\Psi(\eta)=\langle\eta \mid t\rangle$ :

$$
t\left(\eta_{r}\right) \Psi(\eta)=\mathrm{a}\left(\eta_{r}\right) \Psi\left(\eta_{1}, \ldots, q^{-1} \eta_{r}, \ldots, \eta_{\mathrm{N}}\right)+\mathrm{d}\left(\eta_{r}\right) \Psi\left(\eta_{1}, \ldots, q \eta_{r}, \ldots, \eta_{\mathrm{N}}\right),
$$

where $\left(\eta_{1}, \ldots, \eta_{\mathrm{N}}\right) \in \mathbb{B}_{\mathrm{N}}$ with $\mathbb{B}_{\mathrm{N}}$ the set of zeros of the $B$-operator in the SOV representation. Here, the parameter $q$ is a root of unity defined as in (2.6) and (2.7). 
Note that for cyclic representations of an integrable quantum model the set $\mathbb{B}_{\mathrm{N}}$ is a finite subset of $\mathbb{C}^{\mathrm{N}}$. So the coefficients a $\left(\eta_{r}\right)$ and $\mathrm{d}\left(\eta_{r}\right)$ are specified only in a finite number of points where they satisfy the following average value relations 14 :

$$
\mathcal{A}\left(\eta_{r}^{p}\right)=\prod_{k=1}^{p} \mathrm{a}\left(q^{k} \eta_{r}\right), \quad \mathcal{D}\left(\eta_{r}^{p}\right)=\prod_{k=1}^{p} \mathrm{~d}\left(q^{k} \eta_{r}\right) .
$$

Here $\mathcal{A}(\Lambda)$ and $\mathcal{D}(\Lambda)$ are the average values of the operator entries $A(\lambda)$ and $D(\lambda)$ of the monodromy matrix. Let us recall that the operator entries of the monodromy matrix are expected to be polynomials (or Laurent polynomials) in the spectral parameter $\lambda$ so the corresponding average values are polynomials (or Laurent polynomials) in $\Lambda \equiv \lambda^{p}$. It is then natural to introduce the functions $\mathrm{a}(\lambda)$ and $\mathrm{d}(\lambda)$ as polynomial (or Laurent polynomial) solutions of the following average relations:

$$
\mathcal{A}(\Lambda)+\gamma \mathcal{B}(\Lambda)=\prod_{k=1}^{p} \mathrm{a}\left(q^{k} \lambda\right), \quad \mathcal{D}(\Lambda)+\delta \mathcal{B}(\Lambda)=\prod_{k=1}^{p} \mathrm{~d}\left(q^{k} \lambda\right),
$$

where $\mathcal{B}(\Lambda)$ is the average value of the operator $B(\lambda)$ and $\gamma$ and $\delta$ are constant to be fixed.

R2. Let us denote with $Z_{f(\lambda)}$ the set of the zeros of the functions $f(\lambda)$, then:

$$
\exists \lambda_{0} \in Z_{\mathrm{a}(\lambda)}: \lambda_{0} \notin \cup_{h=0}^{2 l-1} Z_{\mathrm{d}\left(\lambda q^{h}\right)} .
$$

R3. The average values of the functions a and d are not coinciding in all the zeros of the $B$-operator:

$$
\mathcal{A}\left(\eta_{a}^{p}\right) \neq \mathcal{D}\left(\eta_{a}^{p}\right) \quad \forall a \in\{1, \ldots,[\mathrm{N}]\} \text { and }\left(\eta_{1}, \ldots, \eta_{[\mathrm{N}]}\right) \in \mathbb{B}_{\mathrm{N}}
$$

The requirement R1 yields the introduction of the $p \times p$ matrix $D(\lambda)$, defined as in (3.21), by the functions $\mathrm{a}(\lambda)$ and $d(\lambda)$ solutions of (6.63). This should allow us to reformulate the spectral problem for the transfer matrix as the problem to classify all the solutions $t(\lambda)$ to the functional equation $\operatorname{det}_{p} D(\Lambda)=0$ in a model dependent class of functions.

The requirement $\mathbf{R} 2$ implies that the rank of the matrix $D(\lambda)$ is almost everywhere $2 l$. Indeed, the condition (6.64) implies $\mathrm{C}_{1, p}\left(\lambda_{0}\right) \neq 0$, independently from the function $t(\lambda)$. Being the cofactor $\mathrm{C}_{1, p}(\lambda)$ a continuous function of the spectral parameter the above statement on the rank of the matrix $D(\lambda)$ follows. Under this condition we can follow the procedure presented in Theorem 2 to construct the solutions of the Baxter equation. Then the self-adjointness of the transfer matrix $T$ allows us to proceed as in section 5 to show the existence of the Q-operator as a function of $\mathrm{T}$.

The requirement $\mathbf{R} 3$ is a sufficient criterion 15 to show the simplicity of the spectrum of $T$ which should imply that the full integrable structure of the quantum model should be described by the

\footnotetext{
${ }^{14}$ The equations in (6.62) are trivial consequences of the SOV representation and of the cyclicity.

${ }^{15}$ It is worth noticing that in the case of the Sine-Gordon model the criterion $\mathbf{R 3}$ does not apply to the representations with $u_{n}=v_{n}=1$. Nevertheless, we have shown the simplicity of $\mathrm{T}$ by using some model dependent properties of the coefficients $\mathrm{a}(\lambda)$ and $\mathrm{d}(\lambda)$, see section 5 of [1].
} 
transfer matrix as soon as the property (B) in definition (1.1) is shown for the model under consideration.

Following the schema here presented, in a future publication we will address the analysis of the spectrum for the so-called $\alpha$-sectors of the Sine-Gordon model (see [1]). The use of this approach is in particular relevant in these sectors of the Sine-Gordon model because a direct construction of the Q-operator leads to some technical difficulty.

\section{A. Properties of the cofactors $\mathbf{C}_{i, j}(\lambda)$}

Let us consider an $M \times M$ tridiagonal matrix 16 :

$$
O \equiv\left(\begin{array}{cccccc}
z_{1} & y_{1} & 0 & \ldots & 0 & 0 \\
x_{1} & z_{2} & y_{2} & 0 & \ldots & 0 \\
0 & x_{2} & z_{3} & y_{3} & & \vdots \\
\vdots & & & \ddots & & \vdots \\
\vdots & & & & \ddots & 0 \\
0 & \ldots & 0 & x_{M-2} & z_{M-1} & y_{M-1} \\
0 & 0 & \ldots & 0 & x_{M-1} & z_{M}
\end{array}\right)
$$

i.e. a matrix with non-zero entries only along the principal diagonal and the next upper and lower diagonals.

Lemma 3. The determinant of a tridiagonal matrix is invariant under the transformation $\varrho_{\alpha}$ which multiplies for $\alpha$ the entries above the diagonal and for $\alpha^{-1}$ the entries below the diagonal leaving the entries on the diagonal unchanged.

Proof. Let us note that the determinant of a tridiagonal matrix admits the following expansion:

$$
\operatorname{det}_{M} O=z_{1} \operatorname{det}_{M-1} O_{1,1}+x_{1} y_{1} \operatorname{det}_{M-2} O_{(1,2),(1,2)},
$$

where we have used the same notations introduced after formula (3.23). By using it, we get that the action of $\varrho_{\alpha}$ reads:

$$
\operatorname{det}_{M} \varrho_{\alpha}(O)=z_{1} \operatorname{det}_{M-1} \varrho_{\alpha}(O)_{1,1}+x_{1} y_{1} \operatorname{det}_{M-2} \varrho_{\alpha}(O)_{(1,2),(1,2)} .
$$

Then the statement follows by induction noticing that the transformation $\varrho_{\alpha}$ leaves always unchanged the determinant of a $2 \times 2$ matrix.

\footnotetext{
${ }^{16} \mathrm{An}$ interesting analysis of the eigenvalue problem for tridiagonal matrices is presented in [65].
} 
Lemma 4. The following properties hold:

$$
\mathrm{C}_{h+i, k+i}(\lambda)=\mathrm{C}_{h, k}\left(\lambda q^{i}\right) \quad \forall i, h, k \in\{1, \ldots, 2 l+1\},
$$

and:

$$
\mathrm{C}_{1,1}(\lambda)=\mathrm{C}_{1,1}(-\lambda) \text { and } \mathrm{C}_{2,1}(\lambda)=q^{\mathrm{N}} \mathrm{C}_{1,2}(-\lambda) \text {. }
$$

Proof. Note that by the definition (4.31) of the cofactors $C_{i, j}(\lambda)$ the equations A.69) are simple consequences of $q^{p}=1$ and are proven exchanging rows and columns in the determinants.

Let us prove now that the cofactor $C_{1,1}(\lambda)=\operatorname{det}_{2 l} D_{1,1}(\lambda)$ is an even function of $\lambda$. The tridiagonality of the matrix $D_{1,1}(\lambda)$ allows us to use the previous lemma:

$$
\begin{aligned}
\mathrm{C}_{1,1}(\lambda) & \equiv \operatorname{det}_{2 l}\left\|t\left(\lambda q^{h}\right) \delta_{h, k}-\mathrm{a}\left(\lambda q^{h}\right) \delta_{h, k+1}-q^{\mathrm{N}} \mathrm{a}\left(-\lambda q^{h+1}\right) \delta_{h, k-1}\right\|_{h>1, k>1} \\
& =\operatorname{det}_{2 l}\left\|t\left(\lambda q^{h}\right) \delta_{h, k}-q^{\mathrm{N}} \mathrm{a}\left(\lambda q^{h}\right) \delta_{h, k+1}-\mathrm{a}\left(-\lambda q^{h+1}\right) \delta_{h, k-1}\right\|_{h>1, k>1} \\
& =\operatorname{det}_{2 l}\left\|t\left(\lambda q^{h}\right) \delta_{h, k}-\mathrm{d}\left(-\lambda q^{k}\right) \delta_{k, h-1}-\mathrm{a}\left(-\lambda q^{k}\right) \delta_{k, h+1}\right\|_{h>1, k>1} \\
& \equiv \operatorname{det}_{2 l}\left(D_{1,1}(-\lambda)\right)^{T}=\mathrm{C}_{1,1}(-\lambda) .
\end{aligned}
$$

To prove now the second relation in A.70 we expand the cofactors:

$$
\begin{aligned}
& \mathrm{C}_{2,1}(\lambda)=\prod_{h=2}^{2 l+1} \mathrm{a}\left(\lambda q^{h}\right)+\mathrm{d}(\lambda) \operatorname{det}_{2 l-1} D_{(1,2),(1,2)}(\lambda), \\
& \mathrm{C}_{1,2}(\lambda)=\prod_{h=1}^{2 l} \mathrm{~d}\left(\lambda q^{h}\right)+\mathrm{a}(\lambda q) \operatorname{det}_{2 l-1} D_{(1,2),(1,2)}(\lambda) .
\end{aligned}
$$

By using the same steps shown in A.71), the tridiagonality of the matrix $\mathrm{D}_{(1,2),(1,2)}(\lambda)$ implies that its determinant is an even function of $\lambda$ from which the statement $\mathrm{C}_{2,1}(\lambda)=q^{\mathrm{N}} \mathrm{C}_{1,2}(-\lambda)$ follows recalling that $\mathrm{d}(\lambda)=q^{\mathrm{N}} \mathrm{a}(-\lambda q)$.

Remark 3. In this article we need only the properties $(\mathrm{A} .70$; ; however, it is worth pointing out that they are special cases of the following properties of the cofactors:

$$
\mathrm{C}_{i, j}(\lambda)=q^{\mathrm{N}(i-j)} \mathrm{C}_{j, i}(-\lambda) \quad \forall i, j \in\{1, \ldots, 2 l+1\} .
$$

The proof of A.74 can be done similarly to that of A.70 but we omit it for simplicity.

Let us use once again the notation $Z_{f}$ for the set of the zeros of a function $f(\lambda)$, then:

Lemma 5. The equations (4.35) and (4.36) imply:

$$
Z_{\mathrm{C}_{1,1}} \cap Z_{\mathrm{C}_{1,2}} \equiv Z_{\mathrm{C}_{1,1}} \cap Z_{\mathrm{C}_{1,2 l+1}}
$$


Proof. The inclusions $\left(Z_{\mathrm{C}_{1,1}} \cap Z_{\mathrm{C}_{1,2}}\right) \backslash Z_{\mathrm{a}} \subset Z_{\mathrm{C}_{1,1}} \cap Z_{\mathrm{C}_{1,2 l+1}}$ and $\left(Z_{\mathrm{C}_{1,1}} \cap Z_{\mathrm{C}_{1,2 l+1}}\right) \backslash Z_{\mathrm{d}} \subset$ $Z_{\mathrm{C}_{1,1}} \cap Z_{\mathrm{C}_{1,2}}$ trivially follow by equation (4.36).

Let us observe now that $\mathrm{C}_{1,2}\left(\lambda q^{-1}\right)$ has no common zero with $\mathrm{a}(\lambda)$ and that $\mathrm{C}_{1,2 l+1}(\lambda q)$ has no common zero with $d(\lambda)$. These statements simply follow from A.73, A.69) and A.72) when we recall that a $(\lambda)$ has no common zero with $\prod_{h=0}^{2 l-1} \mathrm{~d}\left(\lambda q^{h}\right)$ and that $\mathrm{d}(\lambda)$ has no common zero with $\prod_{h=2}^{2 l+1} \mathrm{a}\left(\lambda q^{h}\right)$. So, if $\left(Z_{\mathrm{C}_{1,1}} \cap Z_{\mathrm{C}_{1,2}}\right) \cap Z_{\mathrm{a}}$ is not empty and $\lambda_{0} \in\left(Z_{\mathrm{C}_{1,1}} \cap Z_{\mathrm{C}_{1,2}}\right) \cap Z_{\mathrm{a}}$, the equation (4.35) computed in $\lambda=q^{-1} \lambda_{0}$ implies $\mathrm{C}_{1,2 l+1}\left(\lambda_{0}\right)=0$ being $\mathrm{C}_{1,2}\left(\lambda_{0} q^{-1}\right) \neq 0$, i.e. $\lambda_{0} \in$ $Z_{\mathrm{C}_{1,1}} \cap Z_{\mathrm{C}_{1,2 l+1}}$. Similarly, if $\left(Z_{\mathrm{C}_{1,1}} \cap Z_{\mathrm{C}_{1,2 l+1}}\right) \cap Z_{\mathrm{d}}$ is not empty and $\lambda_{0} \in\left(Z_{\mathrm{C}_{1,1}} \cap Z_{\mathrm{C}_{1,2 l+1}}\right) \cap Z_{\mathrm{d}}$, the equation (4.35) computed in $\lambda=\lambda_{0}$ implies $\mathrm{C}_{1,2}\left(\lambda_{0}\right)=0$ being $\mathrm{C}_{1,2 l+1}\left(\lambda_{0} q\right) \neq 0$, i.e. $\lambda_{0} \in$ $Z_{\mathrm{C}_{1,1}} \cap Z_{\mathrm{C}_{1,2}}$. So that (4.35) implies the inclusions $\left(Z_{\mathrm{C}_{1,1}} \cap Z_{\mathrm{C}_{1,2}}\right) \cap Z_{\mathrm{a}} \subset Z_{\mathrm{C}_{1,1}} \cap Z_{\mathrm{C}_{1,2 l+1}}$ and $\left(Z_{\mathrm{C}_{1,1}} \cap Z_{\mathrm{C}_{1,2 l+1}}\right) \cap Z_{\mathrm{d}} \subset Z_{\mathrm{C}_{1,1}} \cap Z_{\mathrm{C}_{1,2}}$ in this way completing the proof of the lemma.

\section{B. Scalar product in the SOV space}

Here is described as a natural structure of Hilbert space can be provided to the linear space of the SOV representation by preserving the self-adjointness of the transfer matrix.

\section{B.1 Cyclic representations of the Weyl algebra}

Here, we consider the cyclic representations of the Weyl algebra $W_{q}^{(n)}$ in the case:

$$
\mathrm{u}_{n}^{p}=\mathrm{v}_{n}^{p}=1 \text { for } \beta^{2}=p^{\prime} / p \text { with } p^{\prime} \text { even and } p=2 l+1 \text { odd. }
$$

At any site $n$ of the chain, we introduce the quantum space $\mathcal{R}_{n}$ with $\mathrm{v}_{n}$-eigenbasis:

$$
\mathrm{v}_{n}|k, n\rangle=q^{k}|k, n\rangle \forall|k, n\rangle \in \mathrm{B}_{n}=\{|k, n\rangle, \forall k \in\{-l, \ldots, l\}\} .
$$

Note that the eigenvalues of $\mathrm{v}_{n}$ describe the unit circle $\mathbb{S}_{p}=\left\{q^{k}: k \in\{-l, \ldots, l\}\right\}$, indeed $q^{l+1}=$ $q^{-l}$. On $\mathcal{R}_{n}$ is defined a $p$-dimensional representation of the Weyl algebra by setting:

$$
\mathrm{u}_{n}|k, n\rangle=|k+1, n\rangle \forall k \in\{-l, \ldots, l\}
$$

with the cyclicity condition:

$$
|k+p, n\rangle=|k, n\rangle
$$

\section{B.2 Representation in the SOV basis}

The analysis developed in [1] define recursively the eigenbasis $\left\{\left|\bar{\eta}_{1} q^{h_{1}}, \ldots, \bar{\eta}_{\mathrm{N}} q^{h_{\mathrm{N}}}\right\rangle\right\}$ of the $B$ operator in the original representation, i.e. as linear combinations of the elements of the basis $\left\{\left|h_{1}, \ldots, h_{\mathrm{N}}\right\rangle \equiv \bigotimes_{n=1}^{\mathrm{N}}\left|h_{n}, n\right\rangle\right\}$, where $\left|h_{n}, n\right\rangle$ are the elements of the $\mathrm{v}_{n}$-eigenbasis defined in (B.77). To write this change of basis in a matrix form let us introduce the following notations:

$$
\left|y_{j}\right\rangle \equiv\left|\bar{\eta}_{1} q^{h_{1}}, \ldots, \bar{\eta}_{\mathrm{N}} q^{h_{\mathrm{N}}}\right\rangle \text { and }\left|x_{j}\right\rangle \equiv\left|h_{1}, \ldots, h_{\mathrm{N}}\right\rangle
$$


where:

$$
j:=h_{1}+\sum_{a=2}^{\mathrm{N}}(2 l+1)^{(a-1)}\left(h_{a}-1\right) \in\left\{1, \ldots,(2 l+1)^{\mathrm{N}}\right\},
$$

note that this defines a one to one correspondence between N-tuples $\left(h_{1}, \ldots, h_{\mathrm{N}}\right) \in\{1, \ldots, 2 l+1\}^{\mathrm{N}}$ and integers $j \in\left\{1, \ldots,(2 l+1)^{\mathrm{N}}\right\}$, which just amounts to chose an ordering in the elements of the two basis. Under this notation, we have:

$$
\left|y_{j}\right\rangle=\mathrm{W}\left|x_{j}\right\rangle=\sum_{i=1}^{(2 l+1)^{\mathrm{N}}} \mathrm{W}_{i, j}\left|x_{i}\right\rangle
$$

where we are representing $\left|x_{j}\right\rangle$ as the vector $|j\rangle$ in the natural basis in $\mathbb{C}^{(2 l+1)^{\mathrm{N}}}$ and $\mathrm{W}=|| \mathrm{W}_{i, j}||$ is a $(2 l+1)^{\mathrm{N}} \times(2 l+1)^{\mathrm{N}}$ matrix. The matrix $\mathrm{W}$ is defined by recursion in terms of the kernel $K$ constructed in appendix $\mathrm{C}$ of [1], let us use the notation:

$$
K_{\left(\left\{h_{1}, \ldots, h_{\mathrm{N}}\right\}, k_{1},\left\{k_{2}, \ldots, k_{\mathrm{N}}\right\}\right)} \equiv K_{\mathrm{N}}\left(\eta \mid \chi_{2} ; \chi_{\mathbf{1}}\right),
$$

where we are considering the case $\mathrm{N}-\mathrm{M}=1$. Then the recursion reads:

$$
\mathrm{W}_{i, j}^{(\mathrm{N})}=\sum_{k_{2}, \ldots, k_{\mathrm{N}}=1}^{2 l+1} K_{\left(\left\{h_{1}(j), \ldots, h_{\mathrm{N}}(j)\right\}, h_{1}(i),\left\{k_{2}, \ldots, k_{\mathrm{N}}\right\}\right)} \mathrm{W}_{\bar{h}(i), a\left(k_{2}, \ldots, k_{\mathrm{N}}\right)}^{(\mathrm{N}-1)},
$$

where we have introduced the index $(\mathrm{N})$ and $(\mathrm{N}-1)$ in the matrices $\mathrm{W}$ to make clear the step of the recursion. Here, $\left(h_{1}(j), \ldots, h_{\mathrm{N}}(j)\right)$ is the unique N-tuples corresponding to the integer $j \in$ $\left\{1, \ldots,(2 l+1)^{\mathrm{N}}\right\}$ and $h_{1}(i)$ is the first entry in the unique N-tuples corresponding to the integer $i \in\left\{1, \ldots,(2 l+1)^{\mathrm{N}}\right\}$. Moreover, we have defined:

$\bar{h}(i):=1+\frac{i-h_{1}(i)}{2 l+1} \in\left\{1, \ldots,(2 l+1)^{(\mathrm{N}-1)}\right\} \quad$ and $\quad a\left(k_{2}, \ldots, k_{\mathrm{N}}\right)=k_{2}+\sum_{a=3}^{\mathrm{N}}(2 l+1)^{(a-2)}\left(k_{a}-1\right)$,

\section{Remarks:}

a) Under the change of basis $\left\{\left|x_{j}\right\rangle\right\} \rightarrow\left\{\left|y_{j}\right\rangle\right\}$ the generic operator $\mathrm{X}$ transforms for similarity:

$$
\mathrm{X}_{S O V} \equiv \mathrm{W}^{-1} \mathrm{XW}
$$

so from the action of the zero operators $\eta_{a}$ and the shift operators $\mathrm{T}_{a}^{ \pm}$on the $B$-eigenbasis $\left|y_{j}\right\rangle$ :

$$
\eta_{a}\left|y_{j}\right\rangle=\bar{\eta}_{a} q^{h_{a}(j)}\left|y_{j}\right\rangle \text { and } \mathrm{T}_{a}^{ \pm}\left|y_{j}\right\rangle=\left|y_{j \pm(2 l+1)^{(a-1)}}\right\rangle
$$

we have that:

$$
\left(\eta_{a}\right)_{S O V}=\bar{\eta}_{a}\left\|q^{h_{a}(j)} \delta_{i, j}\right\| \text { and }\left(\mathrm{T}_{a}^{ \pm}\right)_{S O V}=\left\|\delta_{i, j \pm(2 l+1)^{(a-1)}}\right\| .
$$


From the above expression we have 17 :

$$
\left(\eta_{a}\right)_{S O V}^{\dagger}=\left(\eta_{a}\right)_{S O V}^{*} \text { and }\left(\mathrm{T}_{a}^{ \pm}\right)_{S O V}^{\dagger}=\left(\mathrm{T}_{a}^{\mp}\right)_{S O V} .
$$

b) The known transformation properties of the entries of the monodromy matrix in the original representation imply:

$$
\left(\begin{array}{ll}
\mathrm{D}_{S O V}(\lambda) & \mathrm{C}_{S O V}(\lambda) \\
\mathrm{B}_{S O V}(\lambda) & \mathrm{A}_{S O V}(\lambda)
\end{array}\right)=\left(\begin{array}{cc}
\mathrm{G}^{-1}\left(\mathrm{~A}_{S O V}\left(\lambda^{*}\right)\right)^{\dagger} \mathrm{G} & -\mathrm{G}^{-1}\left(\mathrm{~B}_{S O V}\left(\lambda^{*}\right)\right)^{\dagger} \mathrm{G} \\
-\mathrm{G}^{-1}\left(\mathrm{C}_{S O V}\left(\lambda^{*}\right)\right)^{\dagger} \mathrm{G} & \mathrm{G}^{-1}\left(\mathrm{D}_{S O V}\left(\lambda^{*}\right)\right)^{\dagger} \mathrm{G}
\end{array}\right)
$$

with $G$ is a positive self-adjoint matrix defined by $G:=W^{\dagger} W$.

c) The quantum determinant relation is invariant under similarity transformations and so we have:

$$
a(\lambda) d\left(\lambda q^{-1}\right)=\mathrm{A}_{S O V}(\lambda) \mathrm{D}_{S O V}\left(\lambda q^{-1}\right)-\mathrm{B}_{S O V}(\lambda) \mathrm{C}_{S O V}\left(\lambda q^{-1}\right),
$$

Lemma 6. The basis $\left\{\left|y_{j}\right\rangle\right\}$ is not an orthogonal basis w.r.t. the natural scalar product on $\left\{\left|x_{j}\right\rangle\right\}$.

Proof. Note that the condition $\left\{\left|y_{j}\right\rangle\right\}$ is an orthogonal basis is equivalent to the statement $\mathrm{G}$ is a diagonal matrix (with positive diagonal entries). Let us recall that the Hermitian conjugation property of $B(\lambda)$ together with the Yang-Baxter commutation relations imply:

$$
\left[\mathrm{B}^{\dagger}(\lambda), \mathrm{B}(\mu)\right]=\left[\mathrm{B}(\mu), \mathrm{C}\left(\lambda^{*}\right)\right]=\frac{q-q^{-1}}{\lambda^{*} / \mu-\mu / \lambda^{*}}\left(\mathrm{~A}\left(\lambda^{*}\right) \mathrm{D}(\mu)-\mathrm{A}(\mu) \mathrm{D}\left(\lambda^{*}\right)\right) \neq 0
$$

that is the operator $B(\lambda)$ is not a normal operator. Now let us show that the non-normality of $B(\lambda)$ implies that $\mathrm{G}$ is not diagonal. Indeed, we can write:

$$
\begin{aligned}
{\left[\mathrm{B}^{\dagger}(\lambda), \mathrm{B}(\mu)\right] } & =\left(\mathrm{W}^{\dagger}\right)^{-1}\left(\mathrm{~B}_{S O V}(\lambda)\right)^{\dagger} \mathrm{GB}_{S O V}(\mu) \mathrm{W}^{-1}-\mathrm{WB}_{S O V}(\mu) \mathrm{G}^{-1}\left(\mathrm{~B}_{S O V}(\lambda)\right)^{\dagger} \mathrm{W}^{\dagger} \\
& =\mathrm{W}\left(\mathrm{G}^{-1}\left(\mathrm{~B}_{S O V}(\lambda)\right)^{\dagger} \mathrm{GB}_{S O V}(\mu)-\mathrm{B}_{S O V}(\mu) \mathrm{G}^{-1}\left(\mathrm{~B}_{S O V}(\lambda)\right)^{\dagger} \mathrm{G}\right) \mathrm{W}^{-1} .
\end{aligned}
$$

Note now that if we assume $\mathrm{G}$ diagonal, then $\mathrm{G}$ commutes both with $\mathrm{B}_{S O V}(\lambda)$ and with $\left(\mathrm{B}_{S O V}(\lambda)\right)^{\dagger}$, being all diagonal matrices in the SOV representation, which implies the absurd $\left[\mathrm{B}^{\dagger}(\lambda), \mathrm{B}(\mu)\right]=$ 0 .

\section{B.3 Scalar product in the SOV space}

The self-adjointness of the family $\mathrm{T}(\lambda)$ implies that the transfer matrix eigenstates are orthogonal under the original scalar product:

$$
\delta_{i, j}=\left(\left|t_{i}\right\rangle,\left|t_{j}\right\rangle\right)
$$

we have chosen the orthonormal ones. Note that the above equation naturally induces a scalar product in the SOV representation obtained under change of basis:

$$
(|b\rangle,|a\rangle)_{S O V} \equiv(G|b\rangle,|a\rangle)
$$

\footnotetext{
${ }^{17}$ Here, we are using the standard notation for the adjoint $X^{\dagger} \equiv\left(X^{*}\right)^{t}$.
} 
that is a scalar product for which the adjoint of a vector $|a\rangle$ is the natural adjoint times the matrix G:

$$
|b\rangle^{\dagger \text { sov }} \equiv\langle b| \mathrm{G} \text { with }\langle b|=\left((|b\rangle)^{t}\right)^{*},
$$

and so for the generic operator $X$ we have:

$$
X^{\dagger S O V} \equiv \mathrm{G}^{-1} X^{\dagger} \mathrm{G}
$$

It is trivial to notice that:

Lemma 7. The family of operators $\mathrm{T}_{S O V}(\lambda)$ is self-adjoint w.r.t. $\dagger_{S O V}$ and the eigenstates $\left|t_{j}\right\rangle_{S O V} \equiv \mathrm{W}^{-1}\left|t_{j}\right\rangle$ are orthonormal w.r.t. the scalar product defined in (B.95). Moreover, it results:

$$
\left(\begin{array}{cc}
\left(\mathrm{A}_{S O V}\left(\lambda^{*}\right)\right)^{\dagger S O V} & \left(\mathrm{~B}_{S O V}\left(\lambda^{*}\right)\right)^{\dagger S O V} \\
\left(\mathrm{C}_{S O V}\left(\lambda^{*}\right)\right)^{\dagger S O V} & \left(\mathrm{D}_{S O V}\left(\lambda^{*}\right)\right)^{\dagger S O V}
\end{array}\right)=\left(\begin{array}{cc}
\mathrm{D}_{S O V}(\lambda) & -\mathrm{C}_{S O V}(\lambda) \\
-\mathrm{B}_{S O V}(\lambda) & \mathrm{A}_{S O V}(\lambda)
\end{array}\right) .
$$

\section{References}

[1] G. Niccoli and J. Teschner, The Sine-Gordon model revisited I, Preprint arXiv: 0910.3173

[2] L.D. Faddeev and L.A. Takhtajan, The quantum method of the inverse problem and the Heisenberg XYZ-model, Russ. Math. Surveys, 34:5 (1979) 1168

[3] P.P. Kulish and E.K. Sklyanin, Quantum inverse scattering method and the Heisenberg ferromagnet, Phys. Lett. A70 (1979) 461-463

[4] L.D. Faddeev, E.K. Sklyanin, L.A. Takhtajan, Quantum inverse problem method: I, Theor. Math. Phys. 57 (1980) 688-706

[5] V.V. Bazhanov, S.L. Lukyanov and A.B. Zamolodchikov, Integrable Structure of Conformal Field Theory, Quantum KdV Theory and Thermodynamic Bethe Ansatz, Commun. Math. Phys. 177 (1996) 381-398.

[6] V.V. Bazhanov, S.L. Lukyanov and A.B. Zamolodchikov, Integrable Structure of Conformal Field Theory II. Q-operator and DDV equation, Commun. Math. Phys. 190 (1997) 247-278.

[7] V.V. Bazhanov, S.L. Lukyanov and A.B. Zamolodchikov, Integrable Structure of Conformal Field Theory III. The Yang-Baxter Relation, Commun. Math. Phys. 200 (1999) 297-324.

[8] A. Antonov and B. Feigin, Quantum Group Representations and Baxter Equation, Phys. Lett. B 392 (1997), 115-122.

[9] M. Rossi and R. Weston, A generalized Q-operator for $U_{q}\left(s l_{2}\right)$ vertex models, J. Phys. A 35 (2002) 10015-10032. 
[10] V.V. Bazhanov, S.L. Lukyanov and A.B. Zamolodchikov, Quantum field theories in finite volume: excited state energies, Nucl. Phys. B 489 (1997) 487-531.

[11] R. Baxter, Eight-vertex model in lattice statistics and one-dimensional anisotropic Heisenberg chain. I. Some fundamental eigenvectors Annals of Physics 76 (1973) 1-24

[12] V.V. Bazhanov, Yu. G. Stroganov, Chiral Potts model as a descendant of the six-vertex model, Journal of Statistical Physics 59 (1990) 799-817

[13] V. Pasquier and M. Gaudin, The periodic Toda chain and a matrix generalization of the Bessel function, J. Phys. A25 (1992) 5243-5252

[14] S.E. Derkachov, Baxters Q-operator for the homogeneous XXX spin chain, J. Phys. A32 (1999) 5299-5316

[15] S.E. Derkachov, G.P. Korchemsky, and A. N. Manashov, Noncompact Heisenberg spin magnets from highenergy QCD. I. Baxter Q-operator and separation of variables, Nucl. Phys. B617 (2001) 375-440; Separation of variables for the quantum $S L(2, \mathbb{R})$ spin chain, JHEP 0307 (2003) 047

[16] L.D. Faddeev and R.M. Kashaev, Quantum dilogarithm, Mod. Phys. Lett. A9 (1994) 427-434

[17] L.D. Faddeev, Discrete Heisenberg-Weyl group and modular group, Lett. Math. Phys. 34 (1995) 249-254

[18] S.N.M. Ruijsenaars, First order analytic difference equations and integrable quantum systems, J. Math. Phys. 38 (1997) 1069-1146

[19] S.L.Woronowicz, Quantum exponential function, Rev. Math. Phys. 12 (2000) 873-920

[20] B. Ponsot and J. Teschner, ClebschGordan and RacahWigner coefficients for a continuous series of representations of $\mathcal{U}_{q}(s l(2, \mathbb{R}))$, Commun. Math. Phys. 224 (2001) 613-655

[21] R.M. Kashaev, The noncompact quantum dilogarithm and the Baxter equations, J. Stat. Phys. 102 (2001) 923-936

[22] R.M. Kashaev, The quantum dilogarithm and Dehn twists in quantum Teichmüller theory, In: Integrable structures of exactly solvable twodimensional models of quantum field theory (Kiev, 2000), 211-221 (NATO Sci.Ser.II Math.Phys.Chem., 35, Kluwer Acad. Publ., Dordrecht, 2001)

[23] A. Bytsko and J. Teschner, R-operator, coproduct and Haar-measure for the modular double of $\mathcal{U}_{q}(s l(2, \mathbb{R}))$, Commun. Math. Phys. 240 (2003) 171196

[24] J. Teschner, Liouville theory revisited, Class. Quant. Grav. 18 (2001) R153-R222; A lecture on the Liouville vertex operators, Int. J. Mod. Phys. A19S2 (2004) 436-458

[25] A.Yu. Volkov, Noncommutative hypergeometry, Commun. Math. Phys. 258 (2005) 257-273 
[26] A. Bytsko, J. Teschner, Quantization of models with non-compact quantum group symmetry. Modular XXZ magnet and lattice sinh-Gordon model, J. Phys. A39 (2006) 12927-12981

[27] H. Bethe, Zur Theorie der Metalle I. Eigenwerte und Eigenfunktionen der linearen Atomkette, Z. Phys. 71 (1931) 205

[28] R.J. Baxter, Exactly Solved Models in Statistical Mechanics, Academic Press, 1982

[29] F.C. Alcaraz, M.N. Barber, M.T. Batchelor, R.J. Baxter and G.R.W. Quispel, Surface exponents of the quantum XXZ, Ashkin-Teller and Potts models, J. Phys. A20 (1987) 6397-6409

[30] N.Yu. Reshetikhin, A Method Of Functional Equations In The Theory Of Exactly Solvable Quantum Systems, Lett. Math. Phys. 7 (1983) 205-213; Sov. Phys. JETP 57 (1983) 691

[31] E.K. Sklyanin, The quantum Toda chain, Lect. Notes Phys. 226 (1985) 196-233

[32] E.K. Sklyanin, Quantum inverse scattering method. Selected topics. In: Quantum groups and quantum integrable systems (World Scientific, 1992) 63-97

[33] E.K. Sklyanin, Separation of variables - new trends, Prog. Theor. Phys. Suppl. 118 (1995) $35-60$

[34] V. Tarasov, Cyclic monodromy matrices for the R-matrix of the six-vertex model and the chiral Potts model with fixed spin boundary conditions. Infinite analysis, Parts A, B (Kyoto, 1991), 963-975, Adv. Ser. Math. Phys., 16, World Sci. Publ., River Edge, NJ, 1992.

[35] A. Klümper, P.A. Pearce, Analytic calculation of scaling dimensions: Tricritical hard squares and critical hard hexagons, J. Stat. Phys. 64 (1991) 13-76

[36] A. Klümper, M. Batchelor, P.A. Pearce, Central charges of the 6- and 19-vertex models with twisted boundary conditions, J. Phys. A23 (1991) 3111-3133

[37] C. Destri, H.J. De Vega, New thermodynamic Bethe ansatz equations without strings, Phys. Rev. Lett. 69 (1992) 2313-2317

[38] C. Destri, H.J. de Vega, Unified Approach to Thermodynamic Bethe Ansatz and Finite Size Corrections for Lattice Models and Field Theories, Nucl. Phys. B438 (1995) 413-454

[39] C. Destri, H.J. de Vega, Non linear integral equation and excited-states scaling functions in the sine-Gordon model, Nucl. Phys. B504 (1997) 621-664

[40] D. Fioravanti, A. Mariottini, E.Quattrini, F. Ravanini, Excited state Destri-De Vega equation for sine-Gordon and restricted sine-Gordon, Phys. Lett. B390 (1997) 243-251

[41] G. Feverati, F. Ravanini, G. Takacs, Truncated conformal space at $c=1$, nonlinear integral equation and quantization rules for multi-soliton states, Phys. Lett. B430 (1998) 264-273 
[42] G. Feverati, F. Ravanini, G. Takacs Nonlinear Integral Equation and Finite Volume Spectrum of Sine-Gordon Theory, Nucl. Phys. B540 (1999) 543-586

[43] G. Feverati, Finite Volume Spectrum of Sine-Gordon Model and its Restrictions, Ph.D. Thesis, Bologna University (2000), hep-th/0001172

[44] F. Ravanini, Finite Size Effects in Integrable Quantum Field Theories, hep-th/0102148

[45] D. Fioravanti, M. Rossi, From the braided to the usual Yang-Baxter relation, J. Phys. A34 (2001) L567-L576

[46] D. Fioravanti, M. Rossi, A Braided Yang-Baxter algebra in a theory of two coupled lattice quantum KdV: Algebraic properties and ABA representations, J. Phys. A35 (2002) 3647-3682

[47] D. Fioravanti, M. Rossi, Exact conserved quantities on the cylinder 1: Conformal case, JHEP 0307:031, (2003)

[48] D. Fioravanti, M. Rossi, Exact conserved quantities on the cylinder. 2. Off critical case, JHEP 0308:042, (2003)

[49] J. Teschner, On the spectrum of the Sinh-Gordon model in finite volume, Nucl.Phys.B799 (2008) 403-429

[50] A.G. Izergin, V.E. Korepin, Lattice versions of quantum field theory models in two dimensions, Nucl. Phys. B205 (1982) 401-413

[51] L.D. Faddeev, A. Yu. Volkov, Hirota Equation as an Example of an Integrable Symplectic Map, Letters in Mathematical Physics 32 (1994) 125-135

[52] V. Bazhanov, A. Bobenko, N. Reshetikhin, Quantum discrete sine-Gordon model at roots of 1: integrable quantum system on the integrable classical background. Comm. Math. Phys. 175 (1996), no. 2, 377-400

[53] V.V. Bazhanov, Chiral Potts model and the discrete Sine-Gordon model at roots of unity, Preprint arXiv:hep-th/0809.2351

[54] V.V. Bazhanov and N.Yu. Reshetikhin, Critical RSOS model and conformal field theory Int. J. Mod. Phys. A4, (1989) 115-142

[55] R.I. Nepomechie, Functional relations and Bethe Ansatz for the XXZ chain, J. Stat. Phys. 111, (2003) 1363-1376

[56] R.I. Nepomechie, Bethe ansatz solution of the open XXZ chain with nondiagonal boundary terms, J. Phys. A37, (2004) 433-440

[57] R.J. Baxter, Superintegrable chiral Potts model: thermodynamic properties, an Inverse model, and a simple associated Hamiltonian, J. Stat. Phys. 57 (1989) 139 
[58] R.J. Baxter, V.V. Bazhanov and J.H.H. Perk, Functional relations for the transfer matrices of the Chiral Potts model, Int. J. Mod. Phys. B4 (1990) 803-869

[59] R.J. Baxter Transfer Matrix Functional Relations for the Generalized $\tau 2(t q)$ Model, J. Stat. Phys. 117 (2004) 1-25

[60] G. von Gehlen, N. Iorgov, S. Pakuliak and V. Shadura, The Baxter-Bazhanov-Stroganov model: separation of variables and the Baxter equation, J. Phys. A: Math. Gen. 39 (2006) 7257-7282

[61] G. von Gehlen, N. Iorgov, S. Pakuliak, V. Shadura and Yu Tykhyy, Form-factors in the BaxterBazhanov-Stroganov model I: norms and matrix elements, J. Phys. A: Math. Theor. 40 (2007) 14117-14138

[62] G. von Gehlen, N. Iorgov, S. Pakuliak, V. Shadura and Yu Tykhyy, Form-factors in the BaxterBazhanov-Stroganov model II: Ising model on the finite lattice, J. Phys. A: Math. Theor. 41 (2008) 095003 (24pp)

[63] M. Gutzwiller, The quantum mechanical Toda lattice II, Ann. of Phys. 133 (1981), 304-331.

[64] S. Kharchev, D. Lebedev, Integral representation for the eigenfunctions of quantum periodic Toda chain, Lett.Math.Phys. 50 (1999) 53-77

[65] V. B. Kuznetsov, E.K. Sklyanin, Eigenproblem for Jacobi matrices: hypergeometric series solution, Phil. Trans. Roy. Soc. Lond. A366 (2008) 1089-1114 